\title{
A Brief Discussion on Problems and Countermeasures of the Vocal Singing Modes in Opera
}

\author{
Farong Zhang \\ 2018 Doctor student of Voice and Music, College of the Music, Hansei University, Gunpo-si, Gyeonggi-do, \\ 435-742, Korea
}

Keywords: Opera, Vocal part, Singing mode, Existing problems, Countermeasures.

\begin{abstract}
There is a long history on the development of opera in China. Chinese songs was explored in the 1930s. The development is not very easy. It evolved from the original single theme to the present diversified theme and to the expression of it by various singing modes. We all know that opera originated in Italy. It expresses the content of the original drama by singing. In the process of singing opera, there is a variety of expressions. This paper discusses the problems existing vocal singing modes in opera from the perspective of the classification and features in opera, and elaborates on the existing problems of the vocal singing modes in opera and some effective measures to the existing problems as well as the future development of opera.
\end{abstract}

\section{The classification and characteristics of the vocal parts in opera singing}

As the appear of Italian opera and bel canto in the 17th century, and the increase of the characters in opera along with the increase of dramatic characteristic, more detailed classification on opera was required to meet the needs of the development of opera. Therefore, It gradually developed to three vocal classification: high, medium, low, which is still used now. And they are also called as high-pitched voice, alto voice and bass line. It also can be classified as soprano, mezzo-soprano, alto, tenor, baritone and bass according to gender. In addition, every vocal parts can be divided to lyric, drama and coloration according to tone and volume. As we know, opera does not only mean to listen to music, but also to express the plot through music. There will be emotional change and ups and downs in the plot, which needs changes in voice and timbre to drive the audience into the plot. So, timbre plays an important role in opera.

\subsection{Female Voice}

\subsubsection{Dramatic soprano, coloratura soprano and lyric soprano}

The soprano can be classified into coloratura soprano and lyric soprano specifically. They have similarities and characteristics. First of all, the sound pattern are generally the same, but they have their own particular emphasis on the vocal parts. The dramatic soprano is the first among their, because dramatic soprano has a higher volume and a higher color concentration, which plays a controlling role mainly. The coloratura soprano emphasizes more on coloratura. The training of coloratura soprano requires to be skillful. The vocal range is wide, and the characteristic is flexible. So, it is called the coloratura soprano. Lyric soprano lay particular stress on lyrical, and the characteristic is emotional. Lyric soprano should be good at expressing the feelings of the characters, which is very important to the drama performance. Lyric soprano can bring emotional appeal to the audience, and take them into the story. It is often expressed by solo performance. And this kind of singing mode is more vivid and appealing.

\subsubsection{Mezzo-soprano}

Mezzo-soprano is also known secondary soprano. The voice of mezzo-soprano always creates a tender and sentimental mood. Mezzo-soprano is not easier than soprano in terms of sound pattern, and the sound pattern is harder to control actually. Mezzo-soprano is a special and rare voice. 
Therefore, as said "It is easy to find soprano, but not a mezzo-soprano". The range of mezzo-soprano is g-g2, even up to b2. The mezzo-soprano can also be divided into higher and lower voice. The mezzo-soprano with lower voice is a real mezzo-soprano, whose tone is close to alto. Among the three tone qualities width, thickness and sonority emphasized in bel canto, mezzo-soprano pays more attention to width and thickness. Mezzo-soprano can soothe the listener's mind with deep, vigorous, euphemistic and charming voice. A good mezzo-soprano can make people addictive. And this is the only reason why mezzo-soprano is considered to be a particularly rare tone.

\subsubsection{Contralto and special contralto}

The contralto is the lowest part of the female voice, and the range is usually from $\mathrm{f}$ of the small group below the central c to $\mathrm{f}$ of the 2nd small group. A contralto doesn't refer to a low voice. It is not as high as a soprano or as valuable as a mezzo-soprano, but it is thick and solid. The expression of the characters' emotion in opera requires the cooperation among tones, and the contralto is indispensable. For example, some characters must be performed by contralto. However, the contralto is used less in opera generally. Therefore, professional personals of contralto in this field is less than those of soprano and mezzo-soprano. Special contralto is round and beautiful, and these special voices can sometimes be disguised as male voice. For example, the character of ratmir in Rutherland And Lyudmila, he needs to be performed by special contralto.

\subsection{Male voice}

\subsubsection{Dramatic tenors, lyrical tenors and light lyrical tenors}

The characteristic of opera is that it can display the sound of strong male voice. The sound is strong and has unique charm, which plays an important role in the opera performance. The dramatic tenor belongs to the aria of the drama played by the actors in the stage play. It is the voice that can directly shape the image of the characters. Moreover, the voice of the tenor is also very loud, which is often used in the opera stage. The lyric tenor refers to the American voice, and the lyric tenor emphasizes lyric, which is the way to express emotion. Its timbre is very skilled, and the voice of lyric tenor is very beautiful. At the same time, it pays great attention to the three-dimensional sense of resonance. Lyrical tenors can also be subdivided into light lyric tenors, which have a more graceful tone and more delicate character emotions expressed.

\subsubsection{Dramatic baritone and lyric baritone}

First of all, the sound pattern of baritone in the opera is similar. The tone of the baritone in the opera is rich, substantial and passionate. Therefore, the range of its application is also in a low tone region and is extremely powerful, and the range extension is dramatic. Its sound is as powerful as that of a combative warrior about to go to war, and is the most common manifestation of the baritone of the plays. Lyric baritone, on the other hand, attaches more importance to the devotion to emotion. Compared with opera baritone, it is more gentle, but easier than opera baritone to lead the audience into the story.

\subsubsection{Bass and special bass}

The sound quality of the bass is composed and soft, which is suitable for those operas which are rich in emotion and the characters have experienced ups and downs. Excellent bass players include Robertson, Tianqiu Wu, etc. The special bass is very rare, and the sound range is c-d1. The voice is deep, vigorous, solemn, and the sound range is not wide, but the volume is large. It is the lowest voice in vocal music. Voices in this kind are often assigned to high-status roles: god, king (townhouse, RON green), priest, or Abba. Whether it's a basso or a special basso, they are designed to perform a role better, and convey a better effect to people. They are scarce, but they are extremely important and indispensable. 


\section{Problems existing in the vocal singing modes in opera}

Opera is a new cultural form combining music with emotion and drama. For opera performers, there is much to be tested. Therefore, there will be some corresponding problems in the normal practice. A good opera requires not only professional skills, but also the emotion of the performer at that time and the control to the scene.

\subsection{Focusing on the chest resonates but ignoring the head voice in the high position and the head cavity resonates}

Opera performers often practice their alto voices by focusing on the chest and ignoring the head voice in the high position and the head cavity resonates. In baritone singing, chest resonance is a must. The rich chest resonance can guarantee the sound plump, firm and powerful. If you do not pay attention to this, you will lose the activity of the voice, so that the expression of emotion is not so incisive and vivid.

\subsection{Wrong phonation of high - pitched and low - pitched areas}

The wrong phonation of high and low voices is also one of the most common problems that opera singers often make when performing. In the training of high position voice, the following aspects should be paid attention: take deep breath; The throat must open, and the larynx should maintain stability; solve the sound area, and do not use the soft beginning sound. However, the low position voice sounds from open singing. There is no vertical and upright sound, and there is no big volume. The sound becomes more and more narrow. That's why you can't sound right.

\subsection{Excessive "pressure throat"}

Pressure throat is simply the pursuit of low throat position, with the forced method to make the position of the larynx lower than the correct position when singing, resulting in the pharyngeal cavity obstruction, and that the larynx is not smooth. It also has to use a lot of breath and physical strength to press the pitch out of the larynx to develop into the singing resonant voice in order to get the resonance. Excessive pressure on the throat, increase the tension of the vocal cords and the intensity of breath, so that the image of the sound is too solid, forming a dramatic resonance illusion. This can cause damage to the throat. Over time.

\subsection{Lack of moderate trills}

In opera, there are trills sometimes in order express emotions more incisively and vividly. In some of today's performances, the performer lacks a moderate vibrato. The length or timing of the vibrato is inappropriate. These will have a certain impact on the performance of the opera, then will make it easy for the audience to get out of the situation, and also make the opera's expression of characters' emotions less vivid.

\section{Effective measures to improve the existing problems of vocal singing in opera}

\subsection{Have good breathing}

It is important to breathe during the performance of an opera. It requires the performer to breathe, and to be able to continue singing slowly. Therefore, in daily life, opera performers should exercise their lung capacity and exercise more. Singing is not only for the voice, but also for breathing and lung capacity, which is what we need to pay attention to when exercising.

\subsection{Enhance the skill-based learning}

We all know that what opera singers have to do is practicing constantly and looking for the state of their voices when they make a sound. In the process of practice, we also know the importance of skill-based learning. Sometimes hard work is not enough. We also need to learn to master skills. Each vocal cord has its own vocal characteristics, we should know how to use it and find the most 
appropriate vocal part. In addition, each high or medium pitch has its own sound characteristics, and we should learn to master the skills, so as to learn and express them in a better way.

\subsection{Focus on emotional expression}

Opera is a combination of emotion and music, in which emotion is a very important element, because it is the most moving. The audience can understand the image of the character in the play according to the emotional changes of the performer, feel the development trend of the story, and then understand the whole story. Audience wii be gradually driven into the opera. This is also a measure to improve the problems in singing, bringing the emotions fully into it and making yourself as the characters in the play. Only in this way can bring appeals to the audience. And the audience can easily resonate with the characters in the play.

\section{The future development of opera}

Chinese opera is in the process of continuous innovation. We all know that opera is a kind of elegant art. In the past, people hardly knew to watch operas or what opera intended to express. With the continuous development of the times, diversified communication methods have made drama more accessible to people's life. Now opera begins to combine with the film, the most familiar element in people's life, so that people have a deeper understanding of opera. The songs in our country are also in the state of continuous innovation. On the basis of preserving the unique cultural elements, the essence of the west must be learned, so as to develop into a real opera with Chinese characteristics. People have a new understanding of elegant art, and try to let the elegant art close to more people. However, in the process of development, we should not cater to the public taste blindly, which will reduce the inherent characteristics of opera. However, with the development of the whole social economy, the development of Chinese opera has great potential.

\section{Summary}

The opera requires the performer to integrate music with emotion. So when performing, the performer's voice will be changed accidentally. We all know that the singing part of opera is divided into tenor, soprano, mezzo-soprano, baritone, bass and contralto, lyrical sounds. Each singing part has its own characteristics and its own sound modes. Most of the problems existing in the singing modes in opera also belong to the lack of mastery of the phonation method of each part by the performers, which leads to the poor performance of the opera. In this regard, opera performers need more professional training, for example, pay attention to breathing, master skills, practice and improve gradually. Opera will have a great breakthrough in its future development. Now our opera is combined with more and more elements to make continuous innovation and breakthroughs, so in terms of the whole situation, our opera will have a great harvest in the future.

\section{References}

[1] Jiaxiang Shang. Development history of European vocal music, China radio and television press, 2009.

[2] Li Wang. The importance of vocal music classification in vocal music singing, Journal of shandong university of arts, 2008(10).

[3] Lu Zhang. Views on the division of vocal parts in vocal music art, Journal of Huaibei division university, 2013 (04).

[4] Yu Liu "brief discussion on some difficult problems in the division and determination of vocal parts".

[5] Zhen Liu, brief discussion on the characteristics of the singing form of several songs, modern reading phase ii. 